REVIEW ARTICLE

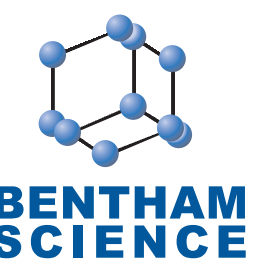

\title{
Sex Differences in Type-2 Diabetes: Implications for Cardiovascular Risk Management
}

Valeria Raparelli ${ }^{1}$, Susanna Morano ${ }^{1}$, Flavia Franconi ${ }^{2}$, Andrea Lenzi ${ }^{1}$ and Stefania Basili ${ }^{3,4, *}$

${ }^{I}$ Department of Experimental Medicine, Sapienza University of Rome, ${ }^{2}$ Department of Biomedical Sciences, University of Sassari, Laboratory of Sex-Gender Medicine, National Institute of Biostructures and Biosystems, Osilo, Italy; ${ }^{3}$ Department of Internal Medicine and Medical Specialties, Sapienza University of Rome, Rome, Italy, ${ }^{4}$ Research Center on Gender and Evaluation and Promotion of Quality in Medicine (CEQUAM), Sapienza-University of Rome, Rome, Italy

\begin{abstract}
Background: Among individuals with Type 2 diabetes (T2DM), cardiovascular disease (CVD) is the leading cause of morbidity and mortality. Sex and gender differences (SGDs) in the cardiovascular consequences of T2DM are relevant suggesting the need for a more aggressive CVD preventive strategy in diabetic women as they lose the so-called "female advantage" in terms of CVD risk comparing with the nondiabetic population. Multiple factors may explain the disproportion in CVD risk among women with diabetes comparing with diabetic men or non-diabetic women. Both genetic and hormonal factors only partially explain SGDs in CVD risk in diabetes. However, women likely reach diagnosis later and in worse conditions, they undergo both diagnostic and therapeutic supports in lower percentage and, finally, they are not able to obtain therapeutic goals recommended by guidelines. Concerning the cardiovascular system, diabetes amplifies the extent of damage at both micro- and macrovascular level differently among sexes.

Methods: The aim of this review is to clarify, in a sex and gender perspective, the impact of diabetes in CVD risk and to summarize the most important SGDs in CVD primary and secondary prevention strategies such as antiplatelet drugs and statins.

Results: The efficacy of ASA and/or statins in secondary prevention is documented in both sexes independently by the presence of T2DM. A different approach to CVD primary prevention with ASA using the age cut-off to discriminate sex differences has been recommended. The use of statins for primary prevention in women should be accurately monitored for the occurrence of myalgia and risk of developing diabetes.
\end{abstract}

\section{A R T I C L E H IS TORY}

Received: December 13, 2016 Accepted: January 27,2017

DOI:

$10.2174 / 1381612823666170130153704$

Conclusion: A gender approach in CVD prevention strategies is urgently required to achieve a sensible reduction of adverse $\mathrm{CV}$ events.

Keywords: Diabetes, antiplatelet therapy, statins, sex, gender, cardiovascular disease, and prevention.

\section{INTRODUCTION}

Cardiovascular disease (CVD) is the worldwide leading cause of morbidity and mortality in both sexes accounting for threequarters of any hospitalization and for half of all deaths [1]

While in the non-diabetic individual, CVD risk is higher in men, type 2 diabetes (T2DM) completely transposes these sexgender differences (SGDs) conferring to women a greater burst of CVD [2]. Interestingly, all risk factors associated with CVD appear to be more aggressive in diabetic females comparing with males [3]. A compromised endothelium-dependent vasodilation, a state of hypercoagulability, worse atherogenic lipid profile, and the coexistence of metabolic syndrome have been reported to be more evident in diabetic women [4-7].

Interestingly, the detrimental effect on cardiovascular (CV) health of an abnormal glycemic control, that defines T2DM, is sexdependent and evident even in pre-diabetic women, that have typically a vascular risk burden increased comparing with the male counterpart [8,9]. Accordingly, the results of the Italian Renal Insufficiency And Cardiovascular Events (RIACE) study showed that in 15,773 patients with T2DM, complications were generally more prevalent in men, but women showed a less favorable CVD risk profile and achieved therapeutic targets to a lesser extent than men, despite the fact that treatment intensity was not lower $[10,11]$.

*Address correspondence to this author at the Department of Internal Medicine and Medical Specialties, Viale del Policlinico 155, 00144 Rome, Italy; E-mail: stefania.basili@uniroma1.it
In a meta-analysis of more than 800,000 subjects, the relative risk of CVD was $44 \%$ higher in women with T2DM than in men [12]. A greater fatality rate after ischemic stroke in women versus men has been reported [13]. Moreover, according to a German and an Italian study, men seem to be paradoxically not prone to have diabetes $[14,15]$.

The increased prevalence of T2DM is concerning in a sex and gender perspective because it confers a higher risk of CV death in women compared with men [16,17]. Specifically, there is a 3 -fold excess in fatal coronary artery disease (CAD) risk in women with T2DM compared with non-diabetic women [17]. Moreover, diabetic women have a 5-fold excess of fatal CAD compared with T2DM men [18]. According to a recent international meta-analysis, a 50-year-old diabetic man has a life expectancy of 5.8 years less than a man of the same age without diabetes; for a 60 -year-old diabetic man, the reduction is by 4.5 years. The corresponding estimates for a woman are 6.4 and 5.4 years, respectively [19]. In the MONICA/KORA study, the risk of myocardial infarction (MI) was four times higher in men and six times higher in women [20].

The reasons for this disproportionate impact of T2DM in females are not entirely clear.

Multifactorial contributions have been suggested such as genetic [21] and inherent physiological differences, including sex hormones impact, differential CVD risk factors prevalence, and SGDs concerning diagnosis and treatment of T2DM and CVD [22].

Therefore, the presence of T2DM represents an imperative for proactive CVD prevention strategies in women. The purpose of this 
review will be to analyze all data available in a sex and gender perspective on CVD prevention in T2DM, specifically targeting the role of antiplatelet and statins therapy.

\subsection{Sex Differences in the Cardiovascular Complications of T2DM: Ischemic Heart Disease}

Globally, contemporary recommendations for the prevention, diagnostic testing, and medical or surgical treatments of ischemic heart disease (IHD) in women are extrapolated from studies conducted predominantly in middle-aged men [23]. When women are enrolled, underrepresentation of female patients in trials of $\mathrm{CV}$ clinical procedures and therapies and inadequate provision of sexspecific analyses limit the ability to define the specific benefits and risks experienced by women [24]. Conversely, in preclinical research, in numerous models of T2DM, male animals are more susceptible to T2DM and have more severe disease than females [24, 25]. Additionally, in some animal models, particularly in the rat, females show less ischemia-reperfusion injury; however, this evidence is not observed in all animal studies [26]. The above findings should indicate that both preclinical and clinical do not take in the right consideration the sex and gender determinants.

Notably, the American Heart Association (AHA) has recently released a position statement on MI in women [27] to highlight the urgent need for a sex- and gender-based personalized approach in CV prevention strategies, to achieve a sensible reduction of adverse $\mathrm{CV}$ events. Therefore, the awareness of sex impact on IHD is progressively increasing and a specific section on diabetes is included. Although men and women share similar risk factors for IHD, several, including T2DM coexistence, are undoubtedly more powerful in women [27].

Of note, diabetic women have a two-fold excess IHD risk compared with men. MI occurs earlier and has higher mortality in women with DM compared with men. An epidemiological study with a long follow-up period showed excess long-term mortality associated with diabetes and excess of $\mathrm{CV}$ disease mortality in diabetic women [28]. Finally, revascularization rates (i.e. angioplasty, coronary artery bypass grafting) are lower in women with T2DM compared with men [23].

In general, the presence of diabetes reduced the so-called female advantage for CVD risk [3]; indeed, mortality from IHD was 3 times higher in women compared with men with diabetes [29].

Multiple factors might contribute to the SGDs in CV consequences of diabetes. The disparities in the female increase of DMrelated CV events could be explained by a differential application of risk factor management strategies or from a differential effect of these management strategies $[30,31]$.

Sex discrepancy in the intensity of CV risk reduction as well as worse hemoglobin A1c control, lower frequency or effects [32] of lipid-lowering therapy, lower aspirin (ASA) use, and lower blood pressure control have been reported in women [30]. In fact, glycemic control along with correction of concomitant high blood pressure is effective to counteract CVD mortality in diabetic women from middle to older age [33]. In addition, diabetic women have less probability to receive appropriate care after acute coronary syndromes [34]. Revascularization rates of CVD with angioplasty and coronary artery bypass surgery are substandard in women than men with T2DM $[35,36]$

The higher mortality and the lower rates of revascularization procedures in women with T2DM admitted for acute coronary syndrome could be partially due to their worse clinical profile as they are older, more likely diagnosed with Non-ST Elevation MI, and have more commonly multiple co-morbidities than men [33]. All these findings could contribute to higher rate of CVD complications in women with T2DM.

Additionally, metabolic factors may supply to the SGDs in CV consequences of diabetes. Since women have a higher prevalence of obesity than men, sex differences in obesity have the potential to magnify existing sex differences in diabetic CV sequelae [37]. For women $\geq 60$ years of age, the prevalence of obesity reached the $38 \%$. Abdominal adiposity was more strongly associated with CV mortality in women compared with men with T2DM in a Finnish population [38].

Finally, worldwide, gender-related aspects at the individual, the population, and the health system levels contribute to sex disparities in CVD in T2DM [39].

Further aspects of SGDs in CV diabetic complications are represented by a more frequent prevalence of drug side effects in women, in addition to increased resistance to the action of drugs used in the prevention or in the therapy of CVD.

\subsection{Sex Differences in CVD Treatments and Interventions in Diabetes: Focus on Anti-Platelet Drugs for Primary Prevention}

While the benefits of ASA in secondary prevention are well documented in both sexes independently by the presence of T2DM [40], there is general consensus across clinical guidelines that ASA for primary prevention should be highly individualized based on a benefit/risk ratio assessment for each patient [41].

In the general population, ASA therapy seems to more effective on platelet in female though some studies have shown no differences in platelet reactivity after low dose ASA therapy between sexes [42].

In line with these results, a recent meta-analysis of U.S. Preventive Services Task Force including 11 primary prevention trials (more than 118,000 participants of healthy and higher-risk populations, with a follow-up ranging from 3.6 to 10.1 years) [43] shows that ASA therapy at varying doses is associated with reductions of non-fatal MI $(-22 \%)$ and all-cause mortality $(-6 \%)$ with not significant decrease in non-fatal total stroke $(5 \%)$ and CV mortality $(-6 \%)$. Moreover, 8 out of 11 studies $(87,524$ participants), that tested only low-dose ASA (100 mg/daily), showed that low-dose ASA significantly increased the risk of major gastrointestinal bleeding $(+58 \%)$ [44].

Several interventional trials with ASA for primary CVD prevention including subjects of both sexes and with or without T2DM are available. Six of these [45-50], planned in the general population, included also a subgroup analysis in diabetic patients, while 3 trials [51-53] were specifically conducted in patients with diabetes mellitus. Among trials, the follow-up for the occurrence of CVD events ranged from 3 to 10 years and the dosage of ASA varied.

The subjects enrolled were predominantly middle-aged adults, frequently only men, [46-47, 49] and the CVD risk differed widely across trials including very low risk or higher risk patients. According to the available literature, the efficacy of ASA therapy, as the primary preventive strategy for CVD, comparing sexes and specifically in women with T2DM is uncertain. Whereas the Antithrombotic Trialists' (ATT) Collaboration showed a reduction in vascular events in the secondary prevention of MI [40], the use of ASA for the primary prevention of CVD events in women, as well as, in patients with T2DM remains controversial. As reported in a large primary prevention trial, a low dose ASA therapy was associated with a lower risk of stroke but not MI or CVD-death in women [45]. Likewise, in the diabetic subgroup of the Women's Health Study, women who received ASA had a lower risk of ischemic stroke compared with those without T2DM, although this outcome was a secondary endpoint [45].

The Early Treatment Diabetic Retinopathy Study Report (ETDRS) randomized diabetic patients (T1 and T2 DM) to 325-mg ASA twice a day or placebo. No overall benefit was shown in the treatment group, and the strength of the findings appeared more evident in men than in women [51]. The Prevention of Progression of Arterial Disease and Diabetes (POPADAD) study, a randomized prevention trial in patients with T2DM and peripheral artery disease 
(PAD), showed no effect of low-dose ASA in prevention of vascular composite endpoints in both sex [52]. Similarly, in the Japanese Primary Prevention of Atherosclerosis with Aspirin for Diabetes (JPAD) trial, evaluated the efficacy of low-dose ASA in diabetic patients without prior CVD, the incidence of composite endpoint was not statistically different between the sexes [53].

Afterward, some meta-analysis, pooling all the available data on primary prevention trials, found evidence for sex-related differences in CV outcomes [54-57]; a larger reduction in CVD events for men and a larger reduction in stroke for women have been observed. In the studies including more than $50 \%$ of women, patients allocated to ASA use had 1.10 relative risk of MI and 0.67 of stroke. Conversely, in trials including more than $50 \%$ of men the relative risk was 0.71 for CVD events and 1.05 for stroke in patients taking ASA [55]. Globally in ASA primary prevention studies, a moderate risk relative reduction (approximately 9\%) for CVD events and relative risk increase of a 2 -fold in bleeding (mainly from the gastrointestinal system) has been recorded.

The net benefit of ASA is influenced in any case by the baseline risks of CVD.

From studies on general middle-aged adults, ASA is highly efficient when the 10-year risk of CVD events is more than $10 \%$ with a baseline low risk of gastrointestinal bleeding [58,59]. This benefit is probably also applicable to T2DM patients; however, more data on sex-specific effects of ASA are needed [60].

Actually, international guidelines [61] suggested a different approach to CVD primary prevention using the age cut-off to discriminate sex differences and a differential approach in management. Low-dose ASA (75-162 mg/d) should be considered for individuals ( $m e n \geq 50$ years of age; women $\geq 60$ years of age) with 10 years CVD risk of at least $10 \%$ who do not have an increased risk of bleeding and have at least 1 additional CVD risk factor. On the contrary, antiplatelet therapy is not suggested for adults (men $<50$ years and women $<60$ years) with diabetes at low risk with no additional CVD risk factors.

\subsection{Sex Differences in CVD Treatments and Interventions in T2DM with Statins}

Statins have dramatically improved the treatment of CV risk. As well as in trials investigating the benefit of antiplatelet drugs in CVD prevention, fewer women are represented in primary and secondary interventional studies with statins [62-64] making it complicated to draw definite conclusions in a sex and gender perspective. The situation is even more difficult in the subgroup of diabetic individuals.

In secondary prevention trials, statins reduce the risk of $\mathrm{CV}$ event to a similar extent in both women and men. In a metaanalysis conducted on data from 26 randomized controlled trials, including more than 170,000 subjects [65], diabetic individuals had a $20 \%$ CV risk reduction in both primary and secondary prevention trials. Women, even if lesser represented (around 2,600 subjects) had a $17 \%$ reduction of $\mathrm{CV}$ events compared with the $23 \%$ in men (approximately more than 10,000) pointing out a statistically significant interaction by sex [65]. According to this meta-analysis, a clear benefit for secondary prevention with statins exists in both women and men, while the evidence for primary prevention of CVD with statins in women is less marked.

Despite that, on the basis of expert opinion and the cholesterol theory of "atheroma progression", the use of statins for primary prevention in women at high risk for CVD is suggested. In fact, coronary atherosclerosis burden was demonstrated to be less in women than in men in the "Study of Coronary Atheroma by Intravascular Ultrasound: Effect of Rosuvastatin Versus Atorvastatin" (SATURN), and atheroma regression, detected by coronary ultrasound, as well as low-density lipoprotein cholesterol (LDL-C) low- ering was higher in female statin users compared with statin-treated males [66].

Accordingly, atheroma regression and LDL lowering is greater with statins in women than in men in the study of coronary atheroma by intravascular ultrasound suggesting that statins may be more beneficial for primary prevention in women, specifically in the ones at high risk for CVD [67].

Statins should be used in subjects with moderate or high CVD risk according to both new American Heart Association/American College of Cardiology (ACC/AHA) guidelines and the Framingham risk calculator [67]. Of note, many more women will susceptible for treatment with statins according to the newer guidelines, which state that all individuals (regardless of sex) $>40$ years of age with T2DM should be treated [67].

Among diabetic population, the approach to statins use seems to be more challenging in a sex and gender perspective. In the Translating Research Into Action for Diabetes (TRIAD) Study [68], women were more likely to have LDL-C levels $<130 \mathrm{mg} / \mathrm{dL}$ and less likely to receive intensive lipid-lowering therapy than men. Therefore, the sex disparity in CVD mortality trends might be related to less aggressive treatment of CVD risk factors in diabetic women [68]. Moreover, in diabetic women, the lack of effects of statins may be also related to an interaction between genetic and environmental factors [32].

According to the current guidelines [67, 69], patients with diabetes mellitus between 40 and 75 years of age without additional atherosclerotic CVD risk factors should be treated with a moderateintensity statin therapy in addition to lifestyle changes (to lower LDL-C on average by $30 \%$ to $50 \%$ ) (ACC/AHA Class I; Level of Evidence A) (ADA Level of Evidence A). High-intensity statin therapy (to lower LDL-C on average by $>50 \%$ ) should be given to diabetics between 40 and 75 years of age with additional atherosclerotic CVD risk factors (ACC/ AHA Class IIa; Level of Evidence B). Among subjects with diabetes mellitus who are $<40$ or $>75$ years of age, the benefit of statin treatment should be individually evaluated (ACC/AHA Class IIa; Level of Evidence C). Interestingly, plasma concentrations of 3-hydroxy-3-methylglutarylCoA (HMG-CoA) reductase inhibitors are generally $15-20 \%$ higher in women than in men, nevertheless, dose adjustments are not necessary. As lipophilic statins, such as simvastatin or atorvastatin, are catalyzed by cytochrome P450 3A4 (CYP3A4), and women have higher concentrations of CYP3A4, women under treatment are more prone to rapidly metabolize these statins [70].

The risk of adverse drug reactions and side effects is greater in women [64]. Among 495 patients $(67 \%$ women) treated with simvastatin/atorvastatin [71], myalgia occurred more frequently in women $(26 \%)$, whereas an increase in creatine phosphokinase or abnormal liver function was more frequent in men $(17.9 \%)$, suggesting the potential for sexual dimorphism in side effects. Besides, these differences have not been explored in larger prospective studies.

As firstly reported by the Justification for the Use of Statins in Prevention: An Intervention Trial Evaluating Rosuvastatin (JUPITER) trial [72], statin-treated women, even if experience a powerful $(44 \%)$ reduction in the risk of major CV events, may have a greater $(25 \%)$ risk of developing diabetes on statins. Thus, a revision to evaluate the incidence of diabetes among 91,140 patients enrolled in thirteen statins interventional trials was performed [73] showing $9 \%$ increased risk for new diagnosis of T2DM, one out of 255 probability of incident diabetes per 4 years of statin use. Moreover, it is suggested that statin-user women had higher probability of incident diabetes [74]. It is reasonable that patients at high risk for T2DM who are treated with statins should be accurately monitored, but statin avoidance is not suggested considering the high benefit in terms of CVD risk decrease [75]. 


\section{CONCLUSION}

Improvements in prevention of CVD and mortality in diabetic patients is challenging. SGDs are clearly evident along the epidemiology, diagnosis and therapeutic response in T2DM patients. Nevertheless, women persist to be scarcely represented in clinical trials compromising the opportunity to test the sex impact on efficacy and safety of the most used CVD prevention strategy, such as antiplatelet drugs and statins. Data available are far from conclusive.

A personalized approach in CVD prevention strategies, considering sex and gender, is urgently required to achieve a sensible reduction of adverse $\mathrm{CV}$ events.

\section{LIST OF ABBREVIATIONS}

$\begin{array}{ll}\text { ASA } & =\text { Aspirin } \\ \text { CAD } & =\text { Coronary artery disease } \\ \text { CYP3A4 } & \text { Cytochrome P450 3A4 } \\ \text { CVD } & =\text { Cardiovascular disease } \\ \text { IHD } & =\text { Ischemic heart disease } \\ \text { LDL-C } & =\text { Low-density lipoprotein cholesterol } \\ \text { MI } & =\text { Myocardial infarction } \\ \text { PAD } & =\text { Peripheral artery disease } \\ \text { SGD } & =\text { Sex-gender difference } \\ \text { T2DM } & =\text { Type 2 diabetes mellitus }\end{array}$

\section{CONFLICT OF INTEREST}

The authors confirm that this article content has no conflict of interest.

\section{ACKNOWLEDGEMENTS}

Declared none.

\section{REFERENCES}

[1] Writing Group Members, Mozaffarian D, Benjamin EJ, Go AS, et $a l$. Heart disease and stroke statistics-2016 update: a report from the american heart association. Circulation 2016; 133: e38-60.

[2] Kannel WB, Wilson PW. Risk factors that attenuate the female coronary disease advantage. Arch Intern Med 1995; 155: 57-61.

[3] $\mathrm{Hu}$ G, Jousilahti P, Qiao Q, Katoh S, Tuomilehto J. Sex differences in cardiovascular and total mortality among diabetic and nondiabetic individuals with or without history of myocardial infarction. Diabetologia 2005; 48: 856-61.

[4] Steinberg HO, Paradisi G, Cronin J, et al. Type II diabetes abrogates sex differences in endothelial function in premenopausal women. Circulation 2000; 101: 2040-46.

[5] Carr ME. Diabetes mellitus: a hypercoagulable state. J Diabetes Complications 2001; 15: 44-54.

[6] Pradhan AD. Sex differences in the metabolic syndrome: implications for cardiovascular health in women. Clin Chem 2014; 60: 44-52.

[7] Oikonomou E, Tsigkou V, Lazaros G, et al. The interaction between gender and diabetes mellitus in the coronary heart disease risk. Curr Pharm Des 2016; 22: 3802-16.

[8] Haffner SM, Miettinen H, Stern MP. Relatively more atherogenic coronary heart disease risk factors in prediabetic women than in prediabetic men. Diabetologia 1997; 40: 711-7.

[9] Donahue RP, Rejman K, Rafalson LB, Dmochowski J, Stranges S, Trevisan M. Sex differences in endothelial function markers before conversion to prediabetes: does the clock start ticking earlier among women? The Western New York Study. Diabetes Care 2007; 30: 354-9.

[10] Pugliese G, Solini A, Bonora E, et al. Chronic kidney disease in type 2 diabetes: lessons from the renal insufficiency and cardiovascular events (RIACE) italian multicentre study. Nutr Metab Cardiovasc $\mathrm{Dis} 2014$; 24 : 815-22.
[11] Penno G, Solini A, Bonora E, et al. Gender differences in cardiovascular disease risk factors, treatments and complications in patients with type 2 diabetes: the RIACE Italian multicentre study. J Intern Med 2013; 274: 176-91.

[12] Huxley R, Barzi F, Woodward M. Excess risk of fatal coronary heart disease associated with diabetes in men and women: metaanalysis of 37 prospective cohort studies. BMJ 2006; 332: 73-8.

[13] Rautio A, Eliasson M, Stegmayr B. Favorable trends in the incidence and outcome in stroke in nondiabetic and diabetic subjects: findings from the Northern Sweden MONICA Stroke Registry in 1985 to 2003 . Stroke $2008 ; 39: 3137-44$

[14] Icks A, Claessen H, Morbach S, Glaeske G, Hoffmann F. Time dependent impact of diabetes on mortality in patients with stroke: survival up to 5 years in a health insurance population cohort in Germany. Diabetes Care 2012; 35: 1868-75.

[15] Policardo L, Seghieri G, Anichini R, et al. Effect of diabetes on hospitalization for ischemic stroke and related in-hospital mortality: a study in Tuscany, Italy, over years 2004-2011. Diabetes Metab Res Rev 2015; 31: 280-6.

[16] Barrett-Connor EL, Cohn BA, Wingard DL, Edelstein SL. Why is diabetes mellitus a stronger risk factor for fatal ischemic heart disease in women than in men? The Rancho Bernardo Study. JAMA 1991; 265: 627-31.

[17] Manson JE, Colditz GA, Stampfer MJ, et al. A prospective study of maturity onset diabetes mellitus and risk of coronary heart disease and stroke in women. Arch Intern Med 1991; 151: 1141-7.

[18] Juutilainen A, Kortelainen S, Lehto S, Rönnemaa T, Pyörälä K, Laakso M. Gender difference in the impact of type 2 diabetes on coronary heart disease risk. Diabetes Care 2004; 27: 2898-904.

[19] Emerging Risk Factors Collaboration, Seshasai SR, Kaptoge S, et al. Diabetes mellitus, fasting glucose, and risk of cause-specific death. N Engl J Med 2011; 364: 829-41.

[20] Icks A, Dickhaus T, Hörmann A, et al. Lower incidence of myocardial infarction in non-diabetic subjects and in diabetic women, but not in diabetic men, in the population aged 25 to 74 years. Findings from the MONICA/KORA myocardial infarction registry in Southern Germany, 1985-2006. Diabetologia 2009; 52: 1836-41.

[21] Paladini F, Adinolfi V, Cocco E, et al. Gender-dependent association of type 2 diabetes with the vasoactive intestinal peptide receptor 1. Gene 2012; 493: 278-81

[22] Gouni-Berthold I, Berthold HK, Mantzoros CS, Böhm M, Krone W. Sex disparities in the treatment and control of cardiovascular risk factors in type 2 diabetes. Diabetes Care 2008; 31: 1389-91.

[23] Garcia M, Mulvagh SL, Bairey Merz CN, Buring JE, Manson JE. Cardiovascular Disease in Women: Clinical Perspectives. Circ Res 2016; 118: 1273-93.

[24] Franconi F, Seghieri G, Canu S, Straface E, Campesi I, Malorni W. Are the available experimental models of type 2 diabetes appropriate for a gender perspective? Pharmacol Res 2008; 57: 6-18.

[25] Islam MS, Loots T. Experimental rodent models of type 2 diabetes: a review. Methods Find Exp Clin Pharmacol 2009; 31: 249-261.

[26] Murphy E, Steenbergen C. Gender-based differences in mechanisms of protection in myocardial ischemia-reperfusion injury. Cardiovasc Res 2007; 75: 478-86.

[27] Mehta LS, Beckie TM, DeVon HA, et al. Acute myocardial infarction in women: a scientific statement from the american heart association. Circulation. 2016; 133: 916-47.

[28] Nedkoff L, Knuiman M, Hung J, Briffa TG. Long-term all-cause and cardiovascular mortality following incident myocardial infarction in men and women with and without diabetes: Temporal trends from 1998 to 2009. Eur J Prev Cardiol 2016; 23: 1273-81.

[29] Gu K, Cowie CC, Harris MI. Diabetes and decline in heart disease mortality in US adults. JAMA 1999; 281: 1291-7.

[30] Wexler DJ, Grant RW, Meigs JB, Nathan DM, Cagliero E. Sex disparities in treatment of cardiac risk factors in patients with type 2 diabetes. Diabetes Care 2005; 28: 514-20.

[31] Chou AF, Scholle SH, Weisman CS, Bierman AS, Correa-deAraujo R, Mosca L. Gender disparities in the quality of cardiovascular disease care in private managed care plans. Womens Health Issues 2007; 17: 120-30

[32] Tavaglione F, Filardi T, Fallarino M, et al. The SNP rs9677 of VPAC1 gene is associated with glycolipid control and heart func- 
tion in female patients with type 2 diabetes: A follow-up study. Nutr Metab Cardiovasc Dis 2016; 26: 109-13.

[33] Puddu PE, Schiariti M, Torromeo C. Gender and cardiovascular mortality in northern and southern european populations. Curr Pharm Des. 2016; 22: 3893-904

[34] Bird CE, Fremont A, Wickstrom S, Bierman AS, McGlynn E. Improving women's quality of care for cardiovascular disease and diabetes: the feasibility and desirability of stratified reporting of objective performance measures. Womens Health Issues 2003; 13: $150-7$.

[35] Vaccarino V, Rathore SS, Wenger NK, et al. Sex and racial differences in the management of acute myocardial infarction, 1994 through 2002. N Engl J Med 2005; 353: 671-82.

[36] Wenger NK. Coronary heart disease in women: highlights of the past 2 years: stepping stones, milestones and obstructing boulders. Nat Clin Pract Cardiovasc Med 2006; 3: 194-202.

[37] Ogden CL, Carroll MD, Kit BK, Flegal KM. Prevalence of childhood and adult obesity in the United States, 2011-2012. JAMA 2014; 311: 806-14

[38] Fredriksson J, Anevski D, Almgren P, et al. Variation in GYS1 interacts with exercise and gender to predict cardiovascular mortality. PLoS One 2007; 2: e285.

[39] Golden SH, Brown A, Cauley JA, et al. Health disparities in endocrine disorders: biological, clinical, and nonclinical factors: an Endocrine Society scientific statement. J Clin Endocrinol Metab 2012; 97: E1579-39.

[40] Antithrombotic Trialists' (ATT) Collaboration, Baigent C, Blackwell $\mathrm{L}$, et al. Aspirin in the primary and secondary prevention of vascular disease: collaborative meta-analysis of individual participant data from randomised trials. Lancet 2009; 373: 1849-60.

[41] Mora S, Manson JE. Aspirin for primary prevention of atherosclerotic cardiovascular disease: advances in diagnosis and treatment. JAMA Intern Med 2016; 176: 1195-204.

[42] Wang TY, Angiolillo DJ, Cushman M, et al. Platelet biology and response to antiplatelet therapy in women: implications for the development and use of antiplatelet pharmacotherapies for cardiovascular disease. J Am Coll Cardiol 2012; 59: 891-900.

[43] Guirguis-Blake JM, Evans CV, Senger CA, O’Connor EA, Whitlock EP, Whitlock EP. Aspirin for the primary prevention of cardiovascular events: a systematic evidence review for the U.S. Preventive Services Task Force. Ann Intern Med 2016; 164: 804-13.

[44] Whitlock EP, Burda BU, Williams SB, Guirguis-Blake JM, Evans $\mathrm{CV}$. Bleeding risks with aspirin use for primary prevention in adults: a systematic evidence review for the U.S. Preventive Services Task Force. Ann Intern Med 2016; 164: 826-35.

[45] Ridker PM, Cook NR, Lee IM, et al. A randomized trial of lowdose aspirin in the primary prevention of cardiovascular disease in women. N Engl J Med 2005; 352: 1293-304.

[46] Peto R, Gray R, Collins R, et al. Randomised trial of prophylactic daily aspirin in British male doctors. Br Med J (Clin Res Ed) 1988; 296: 313-6.

[47] Steering Committee of the Physicians' Health Study Research Group. Final report on the aspirin component of the ongoing Physicians' Health Study. N Engl J Med 1989; 321: 129-35.

[48] Sacco M, Pellegrini F, Roncaglioni MC, et al. Primary prevention of cardiovascular events with low-dose aspirin and vitamin $\mathrm{E}$ in type 2 diabetic patients: results of the Primary Prevention Project (PPP) trial. Diabetes Care 2003; 26: 3264-72.

[49] The Medical Research Council's General Practice Research Framework. Thrombosis prevention trial: randomised trial of lowintensity oral anticoagulation with warfarin and low-dose aspirin in the primary prevention of ischaemic heart disease in men at increased risk. Lancet 1998; 351: 233-41.

[50] Hansson L, Zanchetti A, Carruthers SG, et al. Effects of intensive blood-pressure lowering and low-dose aspirin in patients with hypertension: principal results of the Hypertension Optimal Treatment (HOT) randomised trial. Lancet 1998; 351: 1755-62.

[51] ETDRS Investigators. Aspirin effects on mortality and morbidity in patients with diabetes mellitus. Early Treatment Diabetic Retinopathy Study report 14. JAMA 1992; 268: 1292-300.

[52] Belch J, MacCuish A, Campbell I, et al. Prevention of Progression of Arterial Disease and Diabetes Study Group; Diabetes Registry Group; Royal College of Physicians Edinburgh. The Prevention of
Progression of Arterial Disease and Diabetes (POPADAD) trial: factorial randomised placebo controlled trial of aspirin and antioxidants in patients with diabetes and asymptomatic peripheral arterial disease. BMJ 2008; 337: a1840.

[53] Ogawa H, Nakayama M, Morimoto T, et al. Japanese Primary Prevention of Atherosclerosis With Aspirin for Diabetes (JPAD) Trial Investigators. Low-dose aspirin for primary prevention of atherosclerotic events in patients with type 2 diabetes: a randomized controlled trial. JAMA 2008; 300: 2134-41.

[54] Berger JS, Lala A, Krantz MJ, Baker GS, Hiatt WR. Aspirin for the prevention of cardiovascular events in patients without clinical cardiovascular disease: a meta-analysis of randomized trials. Am Heart J 2011; 162: 115-24

[55] Zhang C, Sun A, Zhang P, et al. Aspirin for primary prevention of cardiovascular events in patients with diabetes: a meta-analysis. Diabetes Res Clin Pract 2010; 87: 211-8.

[56] De Berardis G, Sacco M, Strippoli GF, et al. Aspirin for primary prevention of cardiovascular events in people with diabetes: metaanalysis of randomised controlled trials. BMJ 2009; 339: b4531

[57] Calvin AD, Aggarwal NR, Murad MH, et al. Aspirin for the primary prevention of cardiovascular events: a systematic review and metaanalysis comparing patients with and without diabetes. Diabetes Care 2009; 32: 2300-6.

[58] Pignone M, Earnshaw S, Tice JA, Pletcher MJ. Aspirin, statins, or both drugs for the primary prevention of coronary heart disease events in men: a cost-utility analysis. Ann Intern Med 2006; 144 : 326-36.

[59] Pignone M, Earnshaw S, Pletcher MJ, Tice JA. Aspirin for the primary prevention of cardiovascular disease in women: a costutility analysis. Arch Intern Med 2007; 167: 290-95.

[60] Pignone M, Alberts MJ, Colwell JA, et al. Aspirin for primary prevention of cardiovascular events in people with diabetes: a position statement of the American Diabetes Association, a scientific statement of the American Heart Association, and an expert consensus document of the American College of Cardiology Foundation. Circulation 2010; 121: 2694-701

[61] Regensteiner JG, Golden S, Huebschmann AG, et al. Sex differences in the cardiovascular consequences of diabetes mellitus: a scientific statement from the american heart association. Circulation 2015; 132: 2424-7.

[62] Kern DM, Balu S, Tunceli O, Anzalone D. Statin treatment patterns and clinical profile of patients with risk factors for coronary heart disease defined by National Cholesterol Education Program Adult Treatment Panel III. Curr Med Res Opin 2014; 30: 2443-51.

[63] Ruokoniemi P, Sund R, Arffman M, et al. Are statin trials in diabetes representative of real-world diabetes care: a population-based study on statin initiators in Finland. BMJ Open 2014; 4: e005402.

[64] Rosano GM, Collins P. Gender differences in treatment of cardiovascular disease: a task force on gender of the ESC proposal on gender specific studies in cardiovascular pharmacology. Fundam Clin Pharmacol 2010; 24: 662-3

[65] Cholesterol Treatment Trialists' (CTT) Collaboration, Baigent C, Blackwell L, et al. Efficacy and safety of more intensive lowering of LDL cholesterol: a meta-analysis of data from 170,000 participants in 26 randomised trials. Lancet 2010; 376: 1670-81.

[66] Puri R, Nissen SE, Shao M, et al. Sex-related differences of coronary atherosclerosis regression following maximally intensive statin therapy: insights from SATURN. JACC Cardiovasc Imaging 2014; 7: 1013-22.

[67] Stone NJ, Robinson JG, Lichtenstein AH, et al. 2013 ACC/AHA guideline on the treatment of blood cholesterol to reduce atherosclerotic cardiovascular risk in adults: a report of the American College of Cardiology/American Heart Association Task Force on Practice Guidelines Circulation 2014; 129: S1-S45.

[68] Ferrara A, Mangione CM, Kim C, et al. Sex disparities in control and treatment of modifiable cardiovascular disease risk factors among patients with diabetes: Translating Research Into Action for Diabetes (TRIAD) Study. Diabetes Care 2008; 31: 69-74.

[69] Chamberlain JJ, Rhinehart AS, Shaefer CF Jr, Neuman A. Diagnosis and management of diabetes: synopsis of the 2016 american diabetes association standards of medical care in diabetes. Ann Intern Med 2016; 164: 542-52. 
[70] Soldin OP, Mattison DR. Sex differences in pharmacokinetics and pharmacodynamics. Clin Pharmacokinet 2009; 48: 143-57.

[71] Smiderle L, Lima LO, Hutz MH, et al. Evaluation of sexual dimorphism in the efficacy and safety of simvastatin/atorvastatin therapy in a southern Brazilian cohort. Arq Bras Cardiol 2014; 103: 33-40.

[72] Ridker PM, Danielson E, Fonseca FAH, et al. Rosuvastatin to prevent vascular events in men and women with elevated C-reactive protein. N Engl J Med 2008; 359: 2195-207.
[73] Sattar N, Preiss D, Murray HM, et al. Statins and risk of incident diabetes: a collaborative meta-analysis of randomised statin trials. Lancet 2010; 375: 735-42.

[74] Aiman U, Najmi A, Khan RA. Statin induced diabetes and its clinical implications. J Pharmacol Pharmacother 2014; 5: 181-85.

[75] Goldfine AB. 8Statins: is it really time to reassess benefits and risks? N Engl J Med 2012; 366: 1752-55. 